\section{BMJ Open Respiratory Research}

\title{
Impact of COVID-19 on people with asthma: a mixed methods analysis from a UK wide survey
}

Keir Elmslie James Philip (D) , 1,2,3 Sara Buttery, ${ }^{1,2,3}$ Parris Williams, ${ }^{1,2,3}$ Bavithra Vijayakumar, ${ }^{1,2,3}$ James Tonkin, ${ }^{1,2,3}$ Andrew Cumella, ${ }^{4}$ Lottie Renwick, ${ }^{5}$ Lizzie Ogden, ${ }^{4}$ Jennifer K Quint, ${ }^{1,3,6}$ Sebastian L Johnston, ${ }^{1,2}$ Michael I Polkey, ${ }^{1,2,3}$ Nicholas S Hopkinson (1) 1,2,3

\section{ABSTRACT}

Introduction The impact of acute COVID-19 on people with asthma appears complex, being moderated by multiple interacting disease-specific, demographic and environmental factors. Research regarding longer-term effects in this group is limited. We aimed to assess impacts of COVID-19 and predictors of persistent symptoms, in people with asthma.

Methods Using data from an online UK-wide survey of 4500 people with asthma (median age $50-59$ years, $81 \%$ female), conducted in October 2020, we undertook a mixed methods analysis of the characteristics and experience of those reporting having had COVID-19.

Results The COVID-19 group $(n=471,10.5 \%)$ reported increased inhaler use and worse asthma management, compared with those not reporting COVID-19, but did not differ by gender, ethnicity or household income. Among the COVID-19 group, $56.1 \%$ reported having long COVID, $20.2 \%$ were 'unsure'. Those with long COVID were more likely than those without long COVID to describe: their breathing as worse or much worse after their initial illness ( $73.7 \%$ vs $34.8 \%, p<0.001)$, increased inhaler use $(67.8 \%$ vs $34.8 \%, p<0.001$ ) and worse or much worse asthma management (59.6\% vs $25.6 \%, p<0.001)$. Having long COVID was not associated with age, gender, ethnicity, UK nation or household income.

Analysis of free text survey responses identified three key themes: (1) variable COVID-19 severity, duration and recovery; (2) symptom overlap and interaction between COVID-19 and asthma; (3) barriers to accessing healthcare. Conclusions Persisting symptoms are common in people with asthma following COVID-19. Measures are needed to ensure appropriate healthcare access including clinical evaluation and investigation, to distinguish between COVID-19 symptoms and asthma.

\section{Hospitals, London, UK}

${ }^{4}$ Asthma UK and British Lung Foundation Partnership, London, UK

${ }^{5}$ Policy and External Affairs, Asthma UK, London, UK

${ }^{6}$ Respiratory Epidemiology, Occupational Medicine and Public Health, Imperial College London, London, UK

Correspondence to Dr Keir Elmslie James Philip; k.philip@imperial.ac.uk

\section{INTRODUCTION}

Tens of millions of people have now had COVID-19, with considerable associated morbidity and mortality. As other coronaviruses are a cause of asthma exacerbations, ${ }^{12}$ concerns have been raised regarding the impact of COVID-19 in people with asthma. ${ }^{3}$ Initial efforts to address the

\section{Key messages}

What are the longer-term impacts of COVID-19 on people living with asthma, and which individual characteristics are associated with developing persistent symptoms?

- Persisting COVID-19 symptoms are common in people with asthma. COVID-19 severity, duration and recovery are highly variable, with symptoms frequently overlapping and interacting with their pre-existing respiratory condition.

- To our knowledge this is the first study to use a mixed methods approach to explore the impact of COVID-19 in people with asthma. Although multiple potentially relevant characteristics were investigated, none were associated with the development of long COVID in our population. The study identifies substantial morbidity in this group, including impacts on breathlessness and asthma control, as well as barriers to accessing healthcare and symptom overlap between asthma and persisting COVID-19 symptoms, all with important implications for management.

pandemic have focused on the acute phase of the disease, but the longer-term impacts of the disease have become increasingly apparent under the broad heading of long COVID. ${ }^{45}$ Although an increasing number of studies characterise longer-term impacts of COVID-19 in patients who have been admitted to hospital, the vast majority of people with COVID-19 manage their condition at home. ${ }^{6-8}$ Such individuals have often not been able to access confirmatory laboratory diagnostic testing due to lack of capacity early in the pandemic, and may experience challenges accessing in-depth clinical assessment and characterisation of their condition. ${ }^{9}$

The impact of COVID-19 on people with asthma is complex and likely to vary depending on various factors including the underlying phenotype and severity of their 
asthma, ${ }^{10}$ how well it is controlled, and the effect of medications including inhaled and oral corticosteroids. ${ }^{11} 12$ Some research has suggested that certain factors may confer a degree of protection against COVID-19. ${ }^{13} 14$ However, other studies have suggested people with severe asthma are at an increased risk of death from COVID-19, and asthma increases the risk of requiring critical care among adults admitted to hospital with COVID-19. ${ }^{10} 1516$ Overall, undifferentiated asthma does not appear to be associated with an increased risk of death or hospitalisation due to acute COVID-19. ${ }^{11}$ Research regarding long-term outcomes is limited, though in a population symptom study, asthma was the only pre-existing condition associated with increased risk of developing long COVID. ${ }^{4}$

Improving understanding of how COVID-19 impacts people with asthma, both acutely and longer-term, is vital to ensure ongoing responses to the pandemic are appropriate. ${ }^{17}$ Given these gaps in current research, we conducted a mixed methods analysis focusing on the impacts of COVID-19 in people with asthma.

\section{METHODS}

\section{Study population}

The Asthma UK and British Lung Foundation (AUK-BLF) partnership is conducting UK-wide online surveys periodically to assess the impact of the COVID-19 pandemic and measures to mitigate its spread on people with lung disease. $^{18} 19$ The surveys are distributed via the charity's (AUK-BLF) mailing lists, websites and social media accounts. Previous studies using data from AUK-BLF surveys helped inform the design of this study. ${ }^{18-21}$ For this analysis we used data from the survey conducted in October 2020, and focused on people with self-reported COVID-19.

\section{Statistical analysis}

Quantitative data were analysed using Stata V.14 (StataCorp). In table 1 , data are presented using descriptive statistics. Multiple choice questions were coded as ordinal variables. For the data in table 1, the Kruskal-Wallis test was used to assess for between group differences in the relevant listed variables, defining the groups (dependent variable) by self-reported COVID-19 status (not had COVID-19, not sure if had COVID-19, has had COVID$19)$, which was treated as an ordinal variable. Independent variables all consisted of two or more categories: age groups, gender, ethnicity, care cancelled yes/no, changes to inhaler use, how has been managing asthma. Long COVID status was defined by the response to the question 'Do you think you have had 'long-COVID'?' with the explanation "Long-COVID' is the presence of symptoms (often including fatigue, breathlessness, and muscle aches) for several weeks or months after initially having coronavirus'. In table 2 , logistic regression for ordinal/continuous independent variables, or $\chi^{2}$ tests for binary categorical independent variables, were used as appropriate to assess for differences between those reporting long COVID and those not reporting long COVID (dependent variable) in relation to the demographic and disease-related factors listed. Due to small numbers in some categories, gender was collapsed into a binary variable (male or female); ethnicity to white or all other ethnic groups; age groups ' 17 and under' was collapsed into '29 years and under', and over 80 years collapsed into '70 and above'; nation became a binary variable (England or devolved nations), as did having had a COVID-19 test (positive laboratory test or no positive laboratory test). We used an alpha of $<0.05$ to indicate statistical significance. The $\mathrm{p}$ values presented are not adjusted for multiple comparisons. Readers wanting to adjust for multiple comparisons within each table could apply a Bonferroni alpha of 0.008 ( 0.05 divided by the six variables tested) or 0.006 ( 0.05 divided by eight variables tested) to results in tables 1 and 2, respectively.

\section{Thematic analysis}

We conducted thematic analysis ${ }^{22}$ of free-text responses to the question 'How else would you describe your recovery from coronavirus?'. Initially, these were read and reread for familiarisation and word frequency tables and word clouds were generated NVivo Software (NVivo, 2020) to provide an overview. During the first phases, predominantly open coding was used. These were then refined, using the context from initial readings. Preliminary thematic development was conducted independently by KEJP, SB and PW who then discussed, refined and agreed on the themes. Themes were then reviewed with the other coauthors, with further discussion and reference back to the original data.

\section{Patient and public involvement}

Patients and members of the public were not specifically involved in the design, conduct or reporting of this research. However, as the primary focus of this research was to understand the experience of patients and the content of the survey was informed by the views of patients from previous AUK-BLF surveys.

\section{RESULTS}

\section{Participants}

The survey received 6355 responses (as the survey was publicly accessible, a response rate cannot be reported). Three hundred and nine responses completed on behalf of another person, and 1546 from people who did not have asthma (other long-term respiratory condition or condition not stated) were removed. This left 4500 responses, with $471(10.5 \%)$ from people with selfreported COVID-19 (figure 1).

Of the 4500 total responses, $81 \%$ of respondents were female and median age range was 50-59 years (table 1 ). Gender, ethnicity, household annual income, were similar between those who 'had had', 'had not had' and were 


\begin{tabular}{|c|c|c|c|c|c|}
\hline & $\begin{array}{l}\text { All survey respondents } \\
(n=4500)\end{array}$ & $\begin{array}{l}\text { Not had COVID-19 } \\
(n=3036)\end{array}$ & $\begin{array}{l}\text { Not sure if had COVID-19 } \\
(n=972)\end{array}$ & $\begin{array}{l}\text { Had COVID-19 } \\
(n=471)\end{array}$ & $P$ value \\
\hline Age & $\mathrm{N}(\%)$ & & & & \\
\hline 29 and under & $182(4.09)$ & $122(4.06)$ & $40(4.14)$ & $19(4.09)$ & \\
\hline $30-39$ & $410(9.20)$ & $260(8.65)$ & $95(9.83)$ & $54(11.64)$ & \\
\hline $40-49$ & $891(20.00)$ & $541(18.00)$ & $235(24.33)$ & $112(24.14)$ & \\
\hline $50-59$ & $1270(28.51)$ & $840(27.95)$ & $280(28.99)$ & $142(30.60)$ & \\
\hline $60-69$ & $1085(24.35)$ & $770(25.62)$ & $211(21.84)$ & $101(21.77)$ & \\
\hline 70 and above & $617(13.85)$ & $472(15.71)$ & $105(10.87)$ & $36(7.76)$ & $p<0.001$ \\
\hline \multicolumn{6}{|l|}{ Gender } \\
\hline Female & 3618 (80.79) & $2442(80.86)$ & $778(80.21)$ & $382(81.80)$ & \\
\hline Male & $856(19.12)$ & $577(19.11)$ & $190(19.59)$ & 84 (17.99) & \\
\hline Other & $4(0.09)$ & $1(0.03)$ & $2(0.21)$ & $1(0.21)$ & $p=0.91$ \\
\hline \multicolumn{6}{|l|}{ Ethnicity } \\
\hline White & $4301(96.41)$ & $2909(96.77)$ & $930(96.07)$ & $444(94.67)$ & \\
\hline Black or black British & $37(0.83)$ & $21(0.70)$ & $12(1.24)$ & $4(0.85)$ & \\
\hline Asian or Asian British & $70(1.57)$ & $45(1.50)$ & $16(1.65)$ & $9(1.92)$ & \\
\hline Mixed & $53(1.19)$ & $31(1.03)$ & $10(1.03)$ & $12(2.56)$ & $p=0.28$ \\
\hline \multicolumn{6}{|l|}{ Care cancelled } \\
\hline $\begin{array}{l}\text { Reported that regular care } \\
\text { (eg, an annual review) for } \\
\text { my asthma at the GP has } \\
\text { been cancelled }\end{array}$ & $902(20.04)$ & $613(20.19)$ & $193(19.86)$ & $93(19.75)$ & $p=0.98$ \\
\hline \multicolumn{6}{|c|}{ During lockdown, how has your reliever inhaler use changed? } \\
\hline $\begin{array}{l}\text { I have used it more than } \\
\text { usual }\end{array}$ & $1689(39.96)$ & $1031(35.99)$ & $413(45.64)$ & $238(54.34)$ & \\
\hline $\begin{array}{l}\text { I have used it at about the } \\
\text { same rate }\end{array}$ & $1824(43.15)$ & $1332(46.49)$ & $355(39.23)$ & $128(29.22)$ & \\
\hline $\begin{array}{l}\text { I have used it less than } \\
\text { usual }\end{array}$ & $587(13.89)$ & $409(14.28)$ & $116(12.82)$ & $60(13.70)$ & \\
\hline $\begin{array}{l}\text { I do not have a reliever } \\
\text { inhaler }\end{array}$ & $127(3.00)$ & $93(2.25)$ & $21(2.32)$ & $12(2.74)$ & $\mathrm{p}<0.001$ \\
\hline \multicolumn{6}{|c|}{ Since the start of lockdown, how have you been managing your asthma? } \\
\hline Much worse than usual & $324(7.22)$ & $161(5.32)$ & $84(8.67)$ & $77(16.42)$ & \\
\hline A bit worse than usual & $831(18.52)$ & $502(16.58)$ & $218(22.50)$ & $107(22.81)$ & \\
\hline About the same & $2358(52.44)$ & $1702(56.21)$ & $448(46.23)$ & $197(42.00)$ & \\
\hline A bit better than usual & $584(13.02)$ & $401(13.24)$ & $129(13.31)$ & $51(10.87)$ & \\
\hline Much better than usual & $390(8.69)$ & $262(8.65)$ & $90(9.29)$ & $37(7.89)$ & $p<0.001$ \\
\hline
\end{tabular}

P values are for comparison between groups for people reporting they 'had had', 'had not had' and were 'not sure if they had had' COVID-19.

'not sure if they had had' COVID-19 (table 1). COVID-19 was reported slightly more commonly in younger, than older, age groups, though the median age for each of the COVID-19 status groups was 50-59 years. Twenty per cent of respondents reported having had their regular asthma care cancelled (eg, an annual review at their GP) which was similar between COVID-19 status groupings. 40\% of respondents reported increased reliever inhaler use during lockdown (the first and early part of the second UK wide lock-down), and $26 \%$ of respondents stated they had been managing their asthma 'much worse' or 'a bit worse' (figures 2 and 3). Of note, both increased reliever inhaler use and 'worse' asthma management were more common in those reporting having had COVID-19 (table 1).

\section{Characteristics of population reporting COVID-19}

The population reporting that they had had COVID-19 $(\mathrm{n}=471)$ was $81.8 \%$ female, median age $50-59$ years, $94.7 \%$ white ethnicity, and included a wide age range from all UK nations (England 81\%, Scotland 10.3\%, Wales 6.4\%, Northern Ireland 2.4\%) (table 2). During lockdown, 39.2\% reported their asthma management had worsened, and $54.3 \%$ reported increased inhaler use. 
Table 2 Characteristics of people with asthma who reported having had COVID-19

\begin{tabular}{|c|c|c|c|c|}
\hline & $\begin{array}{l}\text { All with COVID-19 } \\
(n=471)\end{array}$ & $\begin{array}{l}\text { Long COVID } \\
(n=261)\end{array}$ & $\begin{array}{l}\text { Not long COVID } \\
(n=210)\end{array}$ & $P$ value \\
\hline Age & N (\%) & & & \\
\hline 29 and under & 19 (4.09) & $12(4.67)$ & $6(2.99)$ & \\
\hline $30-39$ & $54(11.64)$ & $24(9.34)$ & $28(13.93)$ & \\
\hline $40-49$ & $112(24.14)$ & $65(25.29)$ & 45 (22.39) & \\
\hline $50-59$ & $142(30.60)$ & 78 (30.35) & $64(31.84)$ & \\
\hline 70 and above & $36(7.76)$ & $21(8.17)$ & $15(7.46)$ & $p=0.795$ \\
\hline \multicolumn{5}{|l|}{ Gender } \\
\hline Female & 382 (81.97) & 212 (82.17) & 166 (82.18) & \\
\hline Male & $84(18.03)$ & $46(17.83)$ & $36(17.82)$ & $\mathrm{p}=0.998$ \\
\hline \multicolumn{5}{|l|}{ Total annual household income } \\
\hline Below £20 000 & $116(25.00)$ & $63(24.42)$ & $53(26.50)$ & \\
\hline$£ 20000-£ 30000$ & $90(19.40)$ & $54(20.93)$ & $36(18.00)$ & \\
\hline$£ 30001-£ 40000$ & $53(11.42)$ & $30(11.63)$ & $21(10.50)$ & \\
\hline$£ 40001-£ 70000$ & 74 (15.95) & $41(15.89)$ & $31(15.50)$ & \\
\hline Above $£ 70000$ & $31(6.68)$ & $8(3.10)$ & $21(10.50)$ & \\
\hline Rather not say & $100(21.55)$ & $62(24.03)$ & $38(19.00)$ & $p=0.156$ \\
\hline \multicolumn{5}{|l|}{ Nation } \\
\hline England & $379(80.98)$ & $209(80.38)$ & $167(82.67)$ & \\
\hline \multicolumn{5}{|c|}{ Since the start of lockdown, how have you been managing your asthma? } \\
\hline A bit worse than usual & $107(22.81)$ & $60(23.08)$ & $44(21.67)$ & \\
\hline About the same & $197(42.00)$ & $92(35.38)$ & $104(51.23)$ & \\
\hline A bit better than usual & $51(10.87)$ & $25(9.62)$ & $24(11.82)$ & \\
\hline Much better than usual & $37(7.89)$ & $14(5.38)$ & $23(11.33)$ & $\mathrm{p}<0.001$ \\
\hline \multicolumn{5}{|c|}{ During lockdown, how has your reliever inhaler (usually your blue inhaler) use changed? } \\
\hline I have used it more than usual & $238(54.34)$ & $162(67.78)$ & $75(38.66)$ & \\
\hline I have used it at about the same rate & $128(29.22)$ & $55(23.01)$ & $72(37.11)$ & \\
\hline I have used it less than usual & $60(13.70)$ & $15(6.28)$ & $42(21.65)$ & \\
\hline I do not have a reliever inhaler & $12(2.74)$ & $7(2.93)$ & $5(2.58)$ & $p<0.001$ \\
\hline \multicolumn{5}{|c|}{ Since recovering from coronavirus, breathing described as } \\
\hline Much better than before & $9(1.94)$ & $1(0.39)$ & $8(3.98)$ & \\
\hline Better than before & $7(1.51)$ & $2(0.78)$ & $5(2.49)$ & \\
\hline About the same & $162(34.84)$ & $59(22.87)$ & $101(50.25)$ & \\
\hline Worse than before & $159(34.19)$ & $97(37.60)$ & $60(29.85)$ & \\
\hline Much worse than before & $105(22.58)$ & $93(36.05)$ & $10(4.98)$ & \\
\hline It is too early to say & $23(4.95)$ & $6(2.33)$ & $17(8.46)$ & $p<0.001$ \\
\hline \multicolumn{5}{|l|}{ Have you had long COVID? } \\
\hline Yes & $261(56.13)$ & $261(100)$ & & \\
\hline
\end{tabular}

Continued 


\begin{tabular}{lllll}
\hline Table 2 & Continued & & & \\
\hline & $\begin{array}{l}\text { All with COVID-19 } \\
(\mathbf{n}=\mathbf{4 7 1 )}\end{array}$ & $\begin{array}{l}\text { Long COVID } \\
(\mathbf{n}=\mathbf{2 6 1 )}\end{array}$ & $\begin{array}{l}\text { Not long COVID } \\
(\mathbf{n}=\mathbf{2 1 0})\end{array}$ & P value \\
\hline No & $110(23.66)$ & - & $110(53.92)$ \\
Not sure & $94(20.22)$ & - & $94(46.08)$ \\
\hline
\end{tabular}

Totals of less than 471 are due to non-completion of the question. Not long COVID includes those answering 'no' n=110; 'not sure' $n=94$ or blank $\mathrm{n}=6$. $\mathrm{P}$ values are for univariate logistic regressions (age, income, managing asthma, inhaler use, breathing), or $\chi^{2}$ (gender, ethnicity, nation, positive coronavirus test, having long COVID) tests to assess for differences between those reporting long COVID and those not reporting long COVID.

Fifty-six per cent reported having had long COVID (self-reported), $24 \%$ not, with a further $20 \%$ 'not sure'. Those who reported long COVID were similar to those who reported not having had or being 'unsure' regarding long COVID, for age, gender, ethnicity, UK nation and household income. Compared with those who had not had long COVID and those who were not sure, a higher proportion of individuals who reported that they had long COVID described their breathing as worse or much worse $(73.7 \%$ vs $34.8 \%, \mathrm{p}<0.001)$, an increase in reliever inhaler use $(67.8 \%$ vs $34.8 \%, \mathrm{p}<0.001)$ and that their asthma management was worse or much worse $(59.6 \%$ vs 25.6, $\mathrm{p}<0.001$ ) (table 2) (figures 4-6).

We repeated the analysis with 'not sure' individuals instead classed as having had long COVID, which made no material difference to the results (data not shown).

\section{Patient experience}

Analysis of survey responses revealed three key themes about individuals' experiences following COVID-19 (see figure 7).

1. Variable COVID-19 severity, duration and recovery.

2. Symptom overlap and interaction between COVID-19 and asthma.

3. Barriers to accessing healthcare.

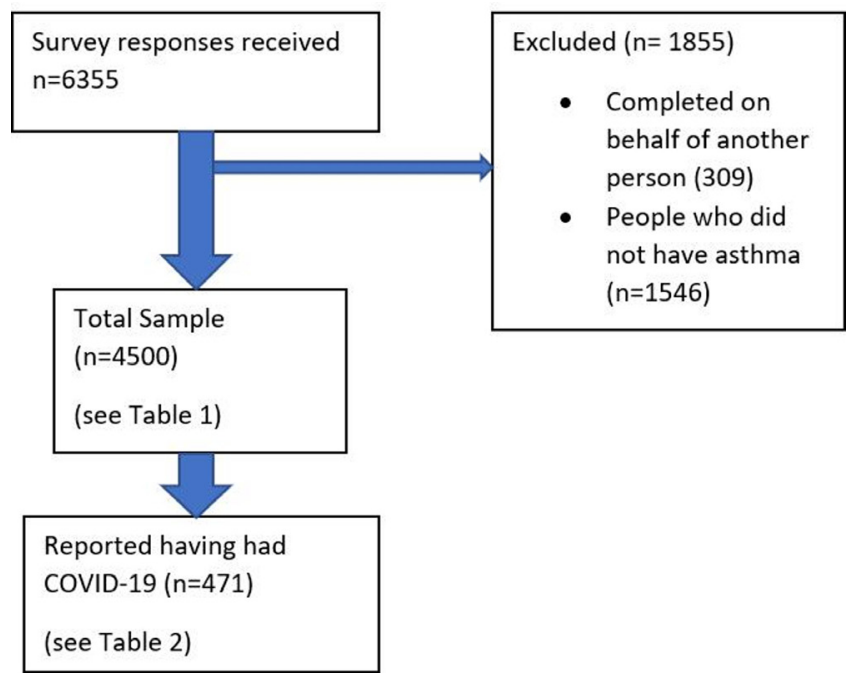

Figure 1 Participant flow diagram.
Theme 1: variable COVID-19 severity, duration and recovery The severity and duration of symptoms described varied considerably between people. Although many reported a rapid recovery after COVID-19 back to their normal state, around half were experiencing ongoing symptoms weeks to many months later. A full spectrum was reported, some had been asymptomatic (detected on screening) or felt that they had had only very mild disease in the acute phase 'Only symptoms I had was loss of taste and smell' (female, aged 30-39), through moderate severity disease, to those requiring intensive care admission.

The trajectory of recovery was also highly variable, with relapsing recoveries being frequently reported, 'slow and unpredictable' (female, aged 40-49) with episodic worsening of symptoms after initial improvements It comes and goes in waves' (female, aged 40-49).

Over half believed that they had long COVID (table 2), with many describing their recovery as 'slow' and 'long'; 'Struggling with Long Covid for 8 months' (female, aged 40-49).

As with severity and trajectory, a wide range of symptoms were reported, however fatigue, breathlessness and pain (often chest, or whole body), were most common,

Walking uphill and upstairs or walking at a faster pace more breathless than before, sometimes feel pulse erratic \& still have discomfort in right lung (female, aged 60-69)

And

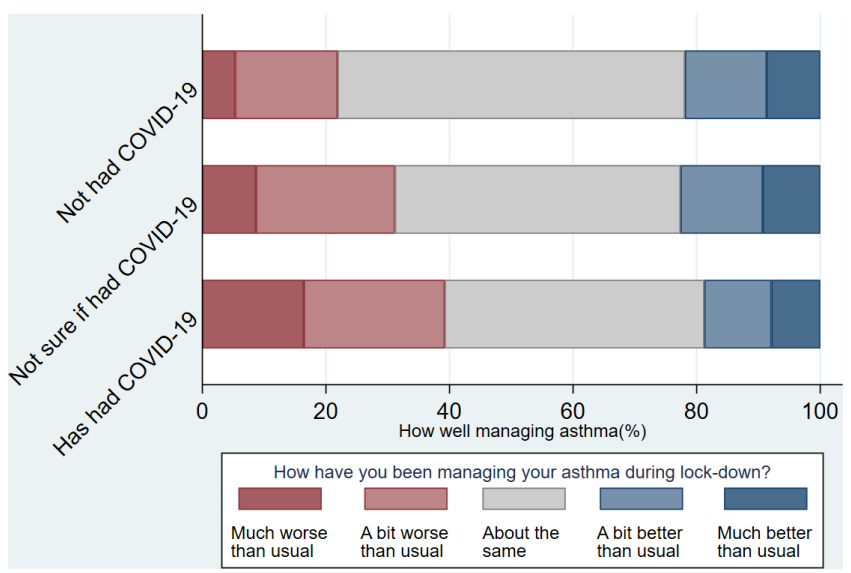

Figure 2 Self-reported asthma management by COVID-19 status. 


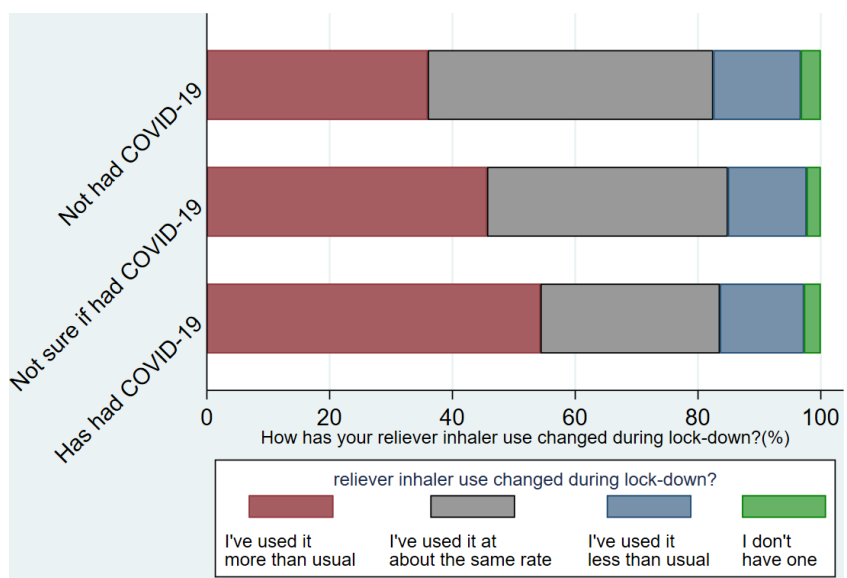

Figure 3 Change in reliever inhaler use by COVID-19 status.

Very draining. Makes day to day living harder. Struggle to do simple things (female, aged 60-69)

Among people with long COVID, some had limited specific symptoms 'Still loss of smell and taste (everything tastes of salt) since March' (female, aged 50-59) while for others the ongoing symptoms were more complex, with major impacts on their activities of daily living and quality of life.

Very, very slow, I was knocked off my feet for six months, from the first week of lockdown and only now feel I have any energy to do things - but my breathing is awful. I used to be able to run for 10 miles, now I can't even manage 10 minutes!! (female, 50-59)

And,

Slow! Still have breathing problems, can't taste or smell. Headaches all the time. Chest pain. Heartburn. Conjunctivitis that doesn't go away. Photophobia. Chronic fatigue. Brain fog. Memory problems. Neuropathy in my legs and feet all worse (female, aged 60-69)

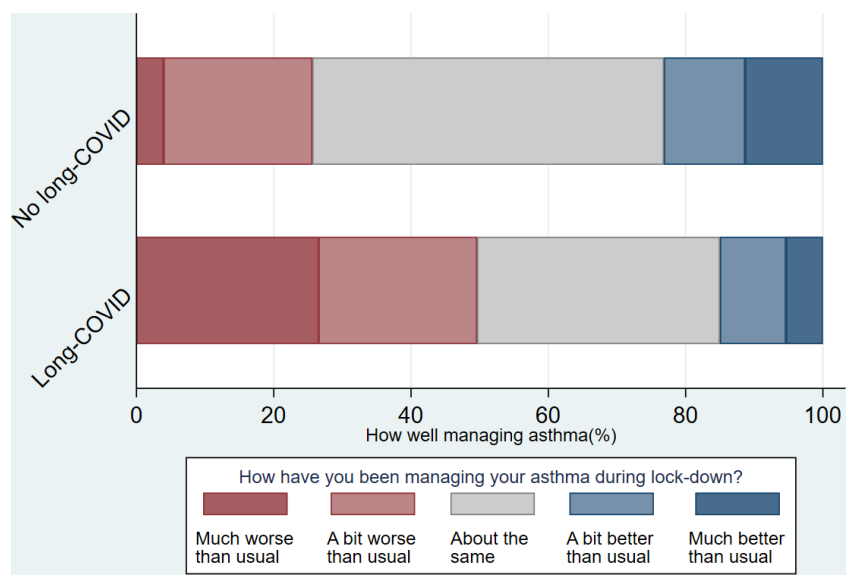

Figure 4 Self-reported asthma management by long COVID status.

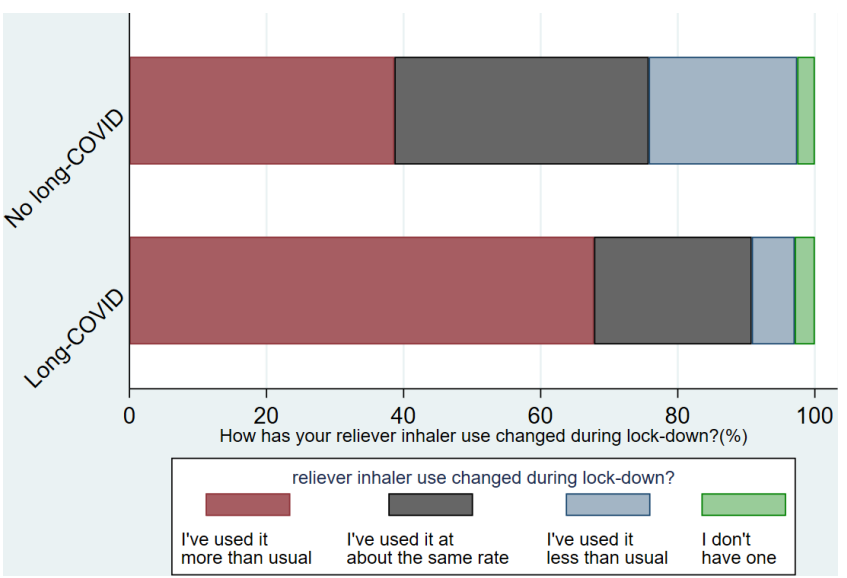

Figure 5 Change in reliever inhaler use by long COVID status.

Many described a disabling combination of physical and psychological impacts.

Very difficult, mentally and physically. I am now on meds for my mental health due to the stress. I lost weight, muscle and general strength, left with a deficiency feeling in my body, as no system has returned to normal since being ill. (female, aged 18-29)

While others gave very positive reports of their recovery, even after very severe disease during the acute phase.

My recovery, once I recovered from the coma has been quite rapid. After 3 months I am able to walk again. I have no cognitive damage, and no pain in my muscles or joints. The one uncertainty is the condition of my lungs, and how much they will improve, as they were badly damaged by the virus. I currently have regular x-rays, and am monitored by a respiratory team to follow how I am healing. (female, aged 60-69)

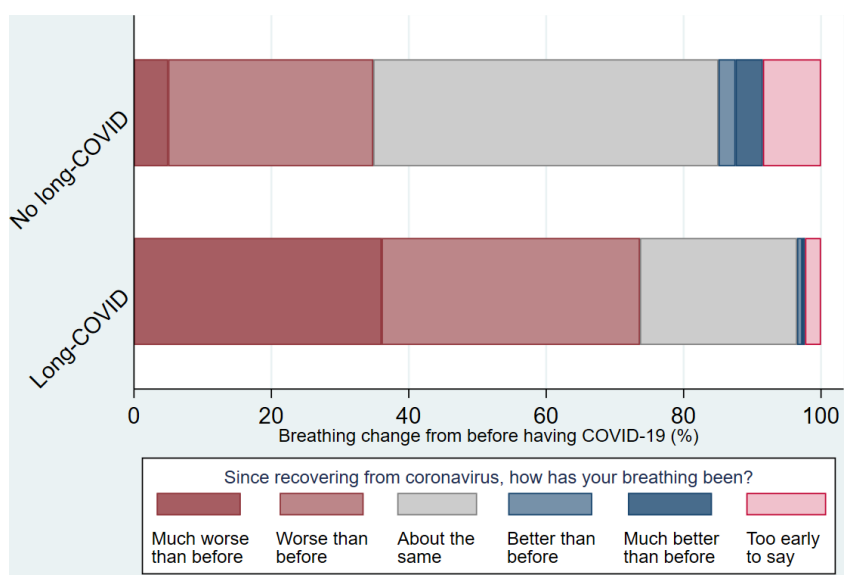

Figure 6 Breathing following COVID-19 by long COVID status. 


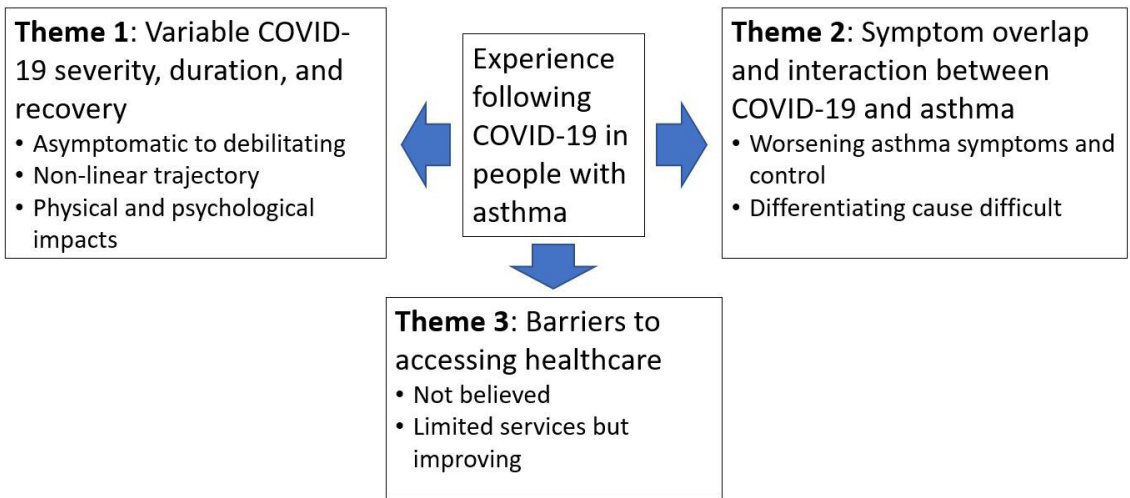

Figure 7 Thematic map.

Theme 2: symptom overlap and interaction between COVID-19 and asthma

Respondents frequently reported that COVID-19 symptoms overlapped with their asthma, with COVID-19 often seen as causing a protracted asthma exacerbation. Many described COVID-19 as causing a worsening of asthma symptoms, but with additional features atypical of their normal condition 'My asthma has changed massively' (female 40-49), and

Usually after a cold I feel my asthma for a week or so, but since being ill in April I have felt my asthma for 6 months-it is getting better though (male, aged 40-49)

And,

I have NEVER had such problems with my asthma or with my oxygen levels. Seven months on, my asthma has still not returned to normal levels and I am still on very high dose of my combined inhaler (female, aged 40-49)

And,

Week 4 recovery and I'm now on daily nebulisers at home and my asthma is much worse with palpitations, fatigue, muscle aches \& headache frightened of the whole situation (female, aged 40-49)

Others saw the COVID-19 and asthma as being distinct, but were not able to decide which was the source of their symptoms 'Unusual in that I get increased breathlessness but don't know whether this is asthma or not' (female, aged 40-49).

In addition, psychological impacts such as anxiety were often mentioned, which added further complexity to the overlapping symptoms of COVID-19 and asthma.

The symptoms have exasperated [sic] my anxiety which in turn affects my asthma (female, aged 50-59)

A small number believed they had COVID-19 earlier than the official first UK cases, describing symptoms consistent with severe respiratory infections that they felt were quite different from previous experiences, 'I am sure I had it in December (2019) as I had all of the symptoms' (female, aged 50-59). And,
I had an unusual serious asthma attack in January it knocked me out for a few weeks, with significant cough with a dry feeling, loss of taste and smell. Pred[nisolone] and antibiotics helped (male, aged 40-49)

\section{Theme 3: barriers to accessing healthcare}

Barriers to accessing and receiving healthcare were commonly reported, especially regarding access to investigations and assessment for COVID-19 related issues. Some felt access to care was limited due to their not 'being believed'; either that they themselves had had COVID-19 or that long COVID was real. Many people who stated they had long COVID, found it challenging to access the care and support they felt would benefit them 'Very long and challenging with little support' (female, aged 30-39) and 'Very long and ongoing issues. No information or help from GP' (female, aged 30-39).

These perceived barriers were a source of distress in themselves,

Was very slow, frustrating and worrying. Did not, and still does not, feel like I was being heard or taken seriously by GPs. Was $6+$ months before I started to see any little bit of improvement in the fatigue and muscle pain. Although better than it was, I am still currently struggling with fatigue and muscle pain. I still feel like I have a constant slight wheeze. Was a real battle getting back into exercising particularly with the breathing issues and had several weeks of that being painful, although I think longer term it did help with the breathing recovery. (female, aged 40-49)

But for some, despite not having confirmatory diagnostic testing earlier in the pandemic, there was a clear perception that healthcare provision for people with long COVID was improving,

I was refused to be tested with extreme shortness of breath and chest pain in March also had upset tummy and burning in my lungs and pain and headaches. 
I've been like this on and off with symptoms since then and the GP thinks it long-COVID but as I was never tested we will never know (female, aged 30-39)

In addition to managing issues attributed to COVID-19 a few people reported disruption to non-COVID-19 related healthcare services '[I] have other ongoing medical problems, operation has been put off' (male, aged 60-69). And, as noted above, $20 \%$ reported that aspects of their usual asthma care had been cancelled.

\section{DISCUSSION}

The results of this large mixed methods study show that people with asthma who have had COVID-19 commonly report increased reliever inhaler use and poorer asthma management. Around half said that they were experiencing long COVID, the occurrence of which was not associated with personal characteristics including age, gender and ethnicity and household income, despite the impact of these on the risk of acute COVID-19. ${ }^{15}$ Individual experience of COVID-19 was highly variable, in terms of severity, symptoms and disease trajectory. COVID-19 symptoms overlapped with, and often exacerbated asthma, leaving individuals struggling to understand their symptoms and how best to manage them.

\section{Significance of findings}

Although a growing body of research exists focusing on the acute phase of COVID-19, longer-term experience of recovery remains less explored. ${ }^{6}$ To our knowledge this is the first study to use a mixed methods approach to explore this in people with asthma. The persisting impact of COVID-19 on breathlessness and asthma control has important implications for management. These findings suggest that people with asthma may be particularly susceptible to protracted symptoms due to COVID-19, though this would require confirmation in further studies including non-asthmatic populations for comparison. Regarding the protracted symptoms reported here, it is unclear to what extent they are due to one of the multiple pathological processes implicated in long COVID, ${ }^{9}$ destabilised asthma, or a combination of factors. Improving access to clinical evaluation and diagnostic services is required to improve understanding and management in this context and the heterogeneity of symptom severities and recovery trajectories underline the need for personalised approaches to assessment and management. The COVID-19 pandemic has led to the suspension of many aspects of routine care for people with long term conditions, including asthma. ${ }^{18} 19$ The reinstatement of regular reviews of inhaler technique and self-management plans as soon as these can be undertaken safely is important, both for asthma control and to help individuals to clarify the source of their symptoms. Online or remote measures to support people with asthma to manage their condition may be of benefit. Because forced expiratory manoeuvres are considered aerosol generating and increase the risk of coronavirus transmission, access to diagnostic tests has become limited. Since breathlessness and variability of symptoms are established features of both long COVID $^{45}$ and of asthma, every effort to distinguish the two is needed to allow appropriate management. It may be that home peak flow monitoring and better access to exhaled nitric oxide (NO) measurement could serve to distinguish the two conditions. Improving access to clinical evaluation and diagnostic services is required to improve understanding and management in this context and the heterogeneity of symptom severities and recovery trajectories underline the need for personalised approaches to assessment and management. ${ }^{23}$

In addition to barriers to healthcare, participants reported distress due to not being believed and not having had an initial diagnostic test to confirm that they had had COVID-19. Most had become ill during the first wave of the pandemic when testing was limited almost entirely to hospitalised cases. This is a challenge for both patients and healthcare professionals, though of note, a positive COVID-19 test is not considered a prerequisite for a diagnosis of long COVID. ${ }^{24}$

\section{Methodological considerations}

Thematic analysis on a large dataset, enabled novel exploratory insights into patient experience not readily achievable through other approaches. The size of the sample, and the range of individuals represented, relative to the typically smaller samples used in qualitative research, means that external validity is increased. However, although this study is based on a large sample, it may not be fully representative of all people with asthma in the UK as a degree of selection bias may exist. In particular, the sample contained a relatively low proportion of men and being online is limited by digital literacy and access. The AUK-BLF surveys tend to have a higher proportion of female respondents which is the likely source of the gender imbalance observed in our sample. Similarly, people with a higher burden of enduring symptoms and those more engaged in their asthma management, may have been more likely to participate. Additionally, data were not collected on when the person had symptoms, so it was not always possible to identify precisely how long they had been present for, although the survey date in October makes it likely that most had become unwell during the first wave in the UK which had passed by the beginning of July 2020 when lockdown measures were eased. A positive antigen or antibody test was not required for inclusion in line with relevant clinical guidelines. ${ }^{17}$ Likewise, the diagnosis of long COVID was based on self-report. As such it is not possible to be completely confident that the prevalence in the sample is representative of all people with asthma in the UK, though of note, of the 4500 people with asthma in the total sample, $471(10.5 \%)$ reported having had COVID-19, which is broadly in line with UK SARS-CoV-2 antibody survey data from the same period. ${ }^{25}$ Furthermore, although adequate 
participant characteristics were collected for the purpose of the study, contextual richness was limited compared with what could have been achieved through other more individualised approaches. More detailed markers of acute COVID-19 severity, and asthma severity would have been useful to aid comparison with other studies as would more in-depth demographic information such as the Index of Multiple Deprivation rather than household income level, but these data were not collected. Also, there could be logical justifications for reporting sex, gender, or both sex and gender, however as self-reported gender was the variable collected by the AUK-BLF survey, this is what has been reported in our study.

\section{CONCLUSION}

For many people with asthma, COVID-19 is associated with prolonged symptoms and worsening asthma control. Experiences post-COVID are highly variable and it can be difficult to distinguish symptoms due to COVID-19 from asthma itself. Difficulties accessing appropriate healthcare and a lack of acknowledgement of patients' condition can both be a source of distress.

Contributors KEJP, SB and PW conducted the analysis. KEJP, AC and NSH verify the underlying data and accept responsibility for the overall content as guarantors. KEJP wrote the first draft of the manuscript. AC, NSH, LR, LO developed the original survey. All authors contributed to the interpretation, reviewing and editing the first draft, and subsequently agreed upon the final manuscript. All authors meet authorship the ICMJE criteria.

Funding KEJP was supported by the Imperial College Clinician Investigator Scholarship. KEJP would like to acknowledge the National Institute for Health Research (NIHR) Biomedical Research Centre based at Imperial College Healthcare NHS Trust and Imperial College London for their support. The views expressed are those of the authors and not necessarily those of the NHS, the NIHR or the Department of Health. SLJ is an NIHR Emeritus Senior Investigator, the Asthma UK Clinical Chair (Grant CH11SJ) and receives support from European Research Council Advanced Grant 788575.

\section{Competing interests None declared.}

Patient and public involvement Patients and/or the public were not involved in the design, or conduct, or reporting, or dissemination plans of this research.

Patient consent for publication Not applicable.

Ethics approval This study involves human participants and was approved by Ethical approval for this study was granted by the Imperial College Research Governance and Integrity Team (RGIT) (ICREC Ref: 20IC6625). Participants gave informed consent to participate in the study before taking part.

Provenance and peer review Not commissioned; externally peer reviewed.

Data availability statement All data relevant to the study are included in the article or uploaded as supplementary information. All relevant data are included in this publication. No other data will be made available due to patient confidentiality.

Open access This is an open access article distributed in accordance with the Creative Commons Attribution Non Commercial (CC BY-NC 4.0) license, which permits others to distribute, remix, adapt, build upon this work non-commercially, and license their derivative works on different terms, provided the original work is properly cited, appropriate credit is given, any changes made indicated, and the use is non-commercial. See: http://creativecommons.org/licenses/by-nc/4.0/.

ORCID iDs

Keir Elmslie James Philip http://orcid.org/0000-0001-9614-3580

Nicholas S Hopkinson http://orcid.org/0000-0003-3235-0454

\section{REFERENCES}

1 Johnston SL, Pattemore PK, Sanderson G, et al. Community study of role of viral infections in exacerbations of asthma in 9-11 year old children. BMJ 1995;310:1225-9.

2 Nicholson KG, Kent J, Ireland DC. Respiratory viruses and exacerbations of asthma in adults. BMJ 1993;307:10.1136/ bmj.307.6910.982:982-6.

3 Johnston SL. Asthma and COVID-19: is asthma a risk factor for severe outcomes? Allergy 2020;75:1543-5.

4 Sudre $\mathrm{CH}$, Murray B, Varsavsky T, et al. Attributes and predictors of long COVID. Nat Med 2021;27:626-31.

5 Greenhalgh T, Knight M, A'Court C, et al. Management of post-acute covid-19 in primary care. BMJ 2020;370:m3026.

6 Hopkinson NS, Jenkins G, Hart N. COVID-19 and what comes after? Thorax 2021;76:324-5.

7 Reilev M, Kristensen KB, Pottegård A, et al. Characteristics and predictors of hospitalization and death in the first 11122 cases with a positive RT-PCR test for SARS-CoV-2 in Denmark: a nationwide cohort. Int J Epidemiol 2020;49:1468-81.

8 Lochlainn MN, Lee KA, Sudre CH. Key predictors of attending hospital with COVID19: an association study from the COVID symptom Tracker APP in 2,618,948 individuals. medRxiv 2020:2020.04.25.20079251.

9 NIHR. Living with Covid19 - second review 2021.

10 Zhu Z, Hasegawa K, Ma B, et al. Association of asthma and its genetic predisposition with the risk of severe COVID-19. J Allergy Clin Immunol 2020;146:327-9.

11 Sunjaya AP, Allida SM, Di Tanna GL, et al. Asthma and risk of infection, hospitalization, ICU admission and mortality from COVID-19: systematic review and meta-analysis. J Asthma 2021:1-14.

12 Ramakrishnan S, Nicolau DV, Langford B, et al. Inhaled budesonide in the treatment of early COVID-19 (STOIC): a phase 2, open-label, randomised controlled trial. Lancet Respir Med 2021;9:10.1016/ S2213-2600(21)00160-0:763-72.

13 Heffler E, Detoraki A, Contoli M, et al. COVID-19 in severe asthma network in Italy (SANI) patients: clinical features, impact of comorbidities and treatments. Allergy 2021;76:10.1111/ all.14532:887-92.

14 Holt $\mathrm{H}$, Talaei M, Greenig M, et al. Risk factors for developing COVID-19: a population-based longitudinal study (COVIDENCE UK). Thorax 2021. doi:10.1136/thoraxjnl-2021-217487. [Epub ahead of print: 30 Nov 2021].

15 Williamson EJ, Walker AJ, Bhaskaran K, et al. Factors associated with COVID-19-related death using OpenSAFELY. Nature 2020:584:430-6.

16 Bloom Cl, Drake TM, Docherty AB, et al. Risk of adverse outcomes in patients with underlying respiratory conditions admitted to hospital with COVID-19: a national, multicentre prospective cohort study using the ISARIC who clinical characterisation protocol UK. Lancet Respir Med 2021:9:699-711.

17 Sivan M, Taylor S. Nice guideline on long covid. BMJ 2020;371:m4938

18 Philip K, Cumella A, Farrington-Douglas J, et al. Respiratory patient experience of measures to reduce risk of COVID-19: findings from a descriptive cross-sectional UK wide survey. BMJ Open 2020;10:e040951.

19 Philip KEJ, Lonergan B, Cumella A, et al. COVID-19 related concerns of people with long-term respiratory conditions: a qualitative study. BMC Pulm Med 2020;20:319.

20 Philip K, Gaduzo S, Rogers J, et al. Patient experience of COPD care: outcomes from the British lung Foundation patient Passport. BMJ Open Respir Res 2019;6:e000478.

21 Buttery S, Philip KEJ, Williams P, et al. Patient symptoms and experience following COVID-19: results from a UK-wide survey. BMJ Open Respir Res 2021;8:e001075.

22 Braun V, Clarke V. Using thematic analysis in psychology. Qual Res Psychol 2006;3:77-101.

23 The Lancet respiratory M. Personalised medicine for asthma in a post-pandemic world. Lancet Respir Med 2021:9:1.

24 NICE. NICE guideline [NG188] COVID-19 rapid guideline: managing the long-term effects of COVID-19. Version 1.7, 2021.

25 Office for National Statistics. Coronavirus (COVID-19) infection survey, antibody data for the UK: 16 March 2021. UK Government, 2021. 